\title{
reCHERches
}

Culture et histoire dans l'espace roman

$12 \mid 2014$

Empreintes/emprunts : entre forces de conformisation et forces d'innovation

\section{Identidad y huellas de Autor: ¿una cuestión literaria o lingüística?}

\section{Kevin Perromat}

\section{(2) OpenEdition}

\section{Journals}

Edición electrónica

URL: https://journals.openedition.org/cher/7715

DOI: $10.4000 /$ cher.7715

ISSN: 2803-5992

\section{Editor}

Presses universitaires de Strasbourg

\section{Edición impresa}

Fecha de publicación: 7 julio 2014

Paginación: 131-145

ISBN: 978-2-86820-569-8

ISSN: 1968-035X

\section{Referencia electrónica}

Kevin Perromat, «Identidad y huellas de Autor: ¿una cuestión literaria o lingüística?», reCHERches [En línea], 12 | 2014, Publicado el 13 diciembre 2021, consultado el 15 diciembre 2021. URL: http:// journals.openedition.org/cher/7715 ; DOI: https://doi.org/10.4000/cher.7715

\section{(c) (†) (2)}

Ce(tte) œuvre est mise à disposition selon les termes de la Licence Creative Commons Attribution -

Pas d'Utilisation Commerciale - Partage dans les Mêmes Conditions 4.0 International. 


\title{
Identidad y huellas de Autor: ¿una cuestión literaria o lingüística?
}

\author{
Kevin Perromat \\ Centre d'Etudes Hispaniques d'Amiens, CERCLL - Université de Picardie-Jules Verne
}

\begin{abstract}
Desde el siglo pasado, la noción de intertextualidad es hegemónica en la Crítica y la Teoría literarias. Este desplazamiento de la atención de los investigadores no se produjo sin que se manifestaran resistencias y fricciones desde las nociones tradicionales que privilegiaban lo individual frente a lo social. Venerables conceptos, que hasta entonces se consideraban como imprescindibles, tales como «estilo», «genio» $\mathrm{o}$ «talento individual», se vieron mayoritariamente relegados a favor de modelos hermenéuticos anclados en la dimensión colectiva de los textos; hago referencia a nociones como «discurso», "género», «hibridación», etc. No obstante, hacia el final del siglo pasado, la generalización de las nuevas tecnologías permitió la elaboración y progresiva sofisticación de las herramientas actuales de análisis de corpus. Gracias a estos desarrollos tecnológicos, la Filología y la Lingüística forense han sido capaces de construir sistemas de nociones, estrategias y protocolos que permiten, en principio, identificar con la mayor certeza posible a los locutores o autores de los textos. Ahora bien, este tipo de formalizaciones y de procedimientos parece presuponer unas nociones de autor y de obra que podrían no corresponder con las empleadas habitualmente por la Crítica y la Teoría literarias.

Partiendo de las últimas contribuciones en las distintas disciplinas y de casos límite de las literaturas hispánicas, algunos interrogantes se imponen en el estado de la cuestión. Estos pueden ser agrupados en dos bloques:
\end{abstract}


1. ¿En qué medida o en qué plano entran en conflicto las distintas perspectivas disciplinarias? ¿Se trata de saberes comparables? ¿Tienen el mismo valor epistemológico? ¿Es posible asociarlos? O, por el contrario, ¿existen espacios de exclusión entre ellas?

2. En definitiva, ¿es posible concebir métodos objetivos y científicos para elucidar las huellas y las identidades literarias?

Obviamente, no pretendo proporcionar aquí respuestas ni tan siquiera parciales a estas cuestiones. Mis razones son varias: en primer lugar, por motivos evidentes de tiempo y de espacio; y, en segundo lugar, pero con mayor peso, porque las respuestas a las preguntas que acabo de formular dependen, con toda probabilidad, de las definiciones mismas, colectivas e individuales, de la Literatura o del Lenguaje, debate infinito que desborda ampliamente y sin margen de dudas el objeto de este coloquio. El propósito de mi intervención es, más modestamente, llamar la atención sobre las mencionadas fricciones, que con frecuencia permanecen ocultas por ser enunciadas en ámbitos disciplinarios diferentes, y proporcionar elementos de reflexión sobre los puntos que me parecen más conflictivos.

\section{¿En qué medida o en qué plano entran en conflicto las distintas perspectivas} disciplinarias empleadas en la identificación de huellas y autores?

Ateniéndonos a una perspectiva estrictamente histórica, es relativamente sencillo comprobar que en los sucesivos nacimientos y renacimientos de la Filología ${ }^{1}$, el estudio de la dimensión lingüística acompañaba invariablemente la dimensión estética de los textos. Esto parece ser una característica común al estudio de los textos hasta bien entrado el siglo XX. Así por ejemplo, en la Antigüedad, la Escuela de Alejandría, que permitió la transmisión y preservación del corpus homérico, basó su labor en la expurgación de los textos existentes de añadidos y lecturas espurias. Para discriminar los textos verdaderos de las imitaciones más modernas, se emplearon criterios lingüísticos que comprendían en lo esencial nociones, reconocibles para nosotros, como "arcaísmo», «idiolecto» o «hápax»». La preminencia de los criterios puramente lingüísticos no excluye que se emplearan nociones próximas a las coordenadas estéticas, puesto que una cierta noción de «estilo», de un idiolecto literario, subyace dentro de muchos de los juicios que configuraron, para bien o para mal, pero definitivamente, el aspecto que

1 Para una excelente exposición y reflexión sobre la historia de la Filología, ver de Joaquín Rubio Tovar (2004). 
tienen en la actualidad los textos que atribuimos a Homero; autor del que, por otra parte, dudamos hasta de su existencia fehaciente.

En el Renacimiento, estudios filológicos similares ocuparon un lugar destacado dentro de la actividad de los humanistas. El éxito clamoroso obtenido por Lorenzo Valla en la demostración del carácter falsario de la denominada Donación de Constantino en 1440 tuvo probablemente mucha influencia en la proliferación de expurgaciones, ediciones críticas en las que, una vez más, los criterios estrictamente lingüísticos se aunaban a los juicios estéticos a la hora de dictaminar las mejores lecturas de los Clásicos o de las Escrituras (Grafton 1990). Por otra parte, cabe destacar que en este periodo se produce un ensanchamiento de las definiciones del texto literario. Por primera vez las nociones de influencia, de la imitatio clásica, de estilo entendido como corriente literaria (así la expresión dolce stil nuovo) proveerán de nuevas e ilimitadas ocupaciones a los estudiosos de los textos. Podríamos ejemplificar esta actividad humanística con el que posiblemente sea el mayor monumento de la filología castellana de la época, las Anotaciones a la poesía de Garcilaso de Fernando de Herrera. Como muestra el trabajo de Herrera, el filólogo humanista rastrea infatigablemente las «huellas» de otros textos y autores para mejor aislar y definir la esencia de la voz creadora $y$ original, las inflexiones y rasgos exclusivos, del texto estudiado: un modelo lingüístico y estético para cada autor. Desde este momento, el estudio de los estilos individuales comprenderá necesariamente una confrontación con las tradiciones y las escuelas literarias. Podríamos añadir, sin simplificar en exceso, que esta continúa siendo hoy día una de las actividades primordiales de los filólogos (y estudiantes de Filología) repartidos por toda la geografía académica.

Se me permitirá que, en aras de la brevedad aunque en detrimento de la exhaustividad, escatime la historia de nuestra disciplina hasta el momento fatídico en que se quebró la armonía solidaria entre las dimensiones literaria y lingüística de los estudios filológicos. Lo que se conoció como «la muerte del autor» conllevaba un cuestionamiento radical de las definiciones tradicionales de la Literatura y del Lenguaje, especialmente al relegar el componente individual a los márgenes de la atención académica. «¿Qué importa quién habla?», se preguntaba Michel Foucault. Por primera vez se quebraba la solidaridad entre las distintas disciplinas filológicas. Ciertamente, la ruptura no fue unánime, la aparición de nuevas subdisciplinas con la consiguiente dispersión y especialización, así como la tolerancia tradicional entre las distintas escuelas fueron factores que contribuyeron a ocultar la 
radical divergencia de los axiomas empleados por unos y otros. Por decirlo de alguna manera, los estilistas, seguidores de Spitzer, Croce o Vossler convivieron con, por ejemplo, los «analistas del discurso» en un perfecto, pero también cordial, desacuerdo.

Con frecuencia las nuevas subespecialidades resultaban de cruces interdisciplinarios, así la Sociolingüística, la Paleografía, el Tratamiento Automatizado del Lenguaje o Lingüística computacional, etc. En otras ocasiones, los departamentos y las especialidades provenían de demandas académicas o profesionales, así la Traductología, los Estudios de Género o la Lingüística forense. Ahora bien, el desplazamiento del objeto y de las finalidades de la actividad académica no ha dejado de tener serias consecuencias, imperceptibles en un primer momento, no ya en la metodología sino en los resultados mismos de la actividad investigadora cuando el objeto es la identidad literaria o lingüística de los textos y sus autores. Cuando los filólogos discuten, por ejemplo, sobre la autoría de la Celestina o del Lazarillo, proponen hipótesis verosímiles que son contrarrestadas y corregidas por otras hipótesis al menos igualmente plausibles. Y aunque este método no parece diferir mayormente de las prácticas seguidas, por ejemplo, por la Lingüística forense, es preciso señalar una importante diferencia que afecta a la orientación y al resultado final de las pesquisas. En el primer caso, los filólogos proponen resultados que conservan un grado de incertidumbre, puesto que siempre pueden aparecer nuevos indicios que desbaraten la autoría propuesta y abran nuevas alternativas; estos filólogos (como los historiadores) viven con frecuencia en un mundo inestable donde las verdades son relativas y los saberes se construyen por consenso más que por certezas.

Desde hace dos siglos, una multitud de críticos, entre ellos muchos ilustres, han tratado de demostrar que tras la firma de Shakespeare se escondía en realidad Francis Bacon. En la literatura francesa existe una teoría parecida con Molière y Corneille de protagonistas. Para el licenciado Avellaneda, al igual que sucede con el anónimo autor del Lazarillo, se han barajado los nombres de, entre otros, Gerónimo de Pasamonte, Lope de Vega, Cristóbal Suárez de Figueroa, sin que los estudiosos hayan llegado a ningún consenso estable ${ }^{2}$. A esta inestabilidad de la identidad del autor con respecto a la obra es a lo que alude, con una buena dosis de ironía, Jorge

2 Enrique Suárez Figaredo proporciona un buen resumen de la situación en su artículo (2011: 261-280). 
Luis Borges en el inicio de la secuencia incluida en el volumen El Hacedor, titulada significativamente «Un problema»:

\section{Texto I}

.... Imaginemos que en Toledo se descubre un papel con un texto arábigo y que los paleógrafos lo declaran de puño y letra de aquel Cide Hamete Benengeli de quien Cervantes derivó el Don Quijote. En el texto leemos que el héroe (que, como es fama, recorría los caminos de España, armado con espada y lanza, y desafiaba por cualquier motivo a cualquiera) descubre, al cabo de uno de sus muchos combates, que ha dado muerte a un hombre. En este punto cesa el fragmento; el problema es adivinar, o conjeturar, cómo reacciona don Quijote. (...)

En este fragmento, Borges rebaja la tradicional actividad de los filólogos a un mero pasatiempo sin trascendencia: Como un eco a la pregunta de Foucault, Borges parece responder que no importa que Cide Hamete Benegeli sea el verdadero autor de las aventuras del Quijote. Lo que realmente cuenta es el giro inesperado que provoca la confirmación de su existencia real en las infinitas lecturas de la obra. Evidentemente, Borges no despreciaba ni la filología tradicional ni las nociones de talento individual o estilo; además, fuera de las ficciones especulativas, se comportaba más bien como un autor ortodoxo en el juego de influencias y apropiaciones, a pesar de las afirmaciones paradójicas que parecen negar esta figura tradicional del autor, asociada a una identidad literaria y lingüística precisa (del tipo «todos somos Homero», «toda la literatura es plagio»). Por otra parte, la flexibilidad y eclecticismo permiten a la Crítica y a la Teoría literarias operar con supuestos relativos, o apreciar, por ejemplo, las especulaciones borgeanas tan evocadas por los críticos posmodernistas, y simultáneamente reconocer un estilo propio, reconocible y original a este autor, Borges, que tanto gustaba de imaginar bibliotecas sin autores o con autores intercambiables.

Los lingüistas forenses, por el contrario, deben proporcionar a sus clientes (el Estado o los particulares) dictámenes certeros y probables, aunque para ello deban sacrificar la precavida relatividad de los saberes filológicos. Esto resulta evidente no sólo por la asimilación de las definiciones jurídicas a las filológicas en los axiomas disciplinarios, sino por la composición misma de los equipos docentes. A modo de ejemplo, en el año 2010-2011, la plantilla de profesores de un Curso de postgrado de Detección de plagio ofrecido por una universidad española estaba compuesta por un detective privado, tres especialistas en Lingüística, un especialista en traducción, un informático, 
un funcionario del cuerpo nacional de Policía (experto en identificación forense), un catedrático de Filología Inglesa y un profesor de Derecho ${ }^{3}$.

Existen, sin embargo, contradicciones insolubles entre las nociones jurídicas y filológicas. En efecto, la Literatura (como las restantes artes) sobrepasa el objeto de estudio de la Lingüística forense. Una obra literaria puede violar la propiedad intelectual de otras, sin que por ello los filólogos o los críticos literarios la condenen o la rechacen como objeto de estudio. Es más, movimientos, escuelas y artistas concretos han defendido estéticas y procedimientos alternativos a las nociones imperantes de autoría y propiedad intelectual: los procedimientos y juegos intertextuales asociados a la Posmodernidad, las obras colectivas, el Apropiacionismo, la parodia seria (blank parody), los fándoms -comunidades de fans que continúan y proponen tramas alternativas para sus películas, series y textos favoritos-, el Plagiarismo, el Copyfight, la Postpoesía, el Afterpop... ${ }^{4}$ Todas estas alternativas son incompatibles con las nociones jurídicas de obra y de autor, favorables a las Industrias Culturales y empleadas por la Lingüística forense. Esta se ve obligada a razonar en términos que privilegian el componente individual antes que el colectivo en el proceso de creación, el análisis cuantitativo antes que cualitativo, la noción misma de obra como objeto delimitable y estable (entidad jurídica, objeto de derecho) antes que como un proceso abierto y continuo (la obra dentro de su tradición, como juego de influencias y referencias).

Para que se comprenda mejor este último punto, me serviré de dos textos derivados, dependientes en el juego de intertextualidad y de la imitación. El primero es obra de Ernesto Sabato quien se prestó en 1995 al ejercicio tradicional del pastiche, tomando como modelo el inconfundible estilo de Borges en las páginas del diario español El país. El segundo es un texto prohibido, publicado en 2011 y retirado de la circulación ese mismo año. Su autor, Agustín Fernández Mallo, fundador del movimiento de las Postpoesía, lo había titulado El hacedor (de Borges), Remake. La viuda de Borges consideró -en mi opinión erróneamente- que vulneraba los derechos

3 Se trata de la Universidad Pompeu Fabra de Barcelona, el curso continúa siendo ofrecido por el Institut Universitari de Lingüística Aplicada (IULA). El resultado de la formación es un diploma universitario que acredita como «experto en detección de plagio»; para una crítica de las implicaciones teóricas que permiten este tipo de «acreditación» ver mi tesis doctoral donde trato la cuestión extensamente: El plagio en las literaturas hispánicas: Historia, Teoría y Práctica (2010).

4 Para una exposición de los puntos comunes y divergentes entre estas propuestas contemporáneas ver K. Perromat (2011). 
de autor, de los que es la única titular, y presionó a la editorial española para que retirara la obra de la circulación, a lo que esta accedió sin dilación.

Texto II Ernesto Sabato, «El tetrágono de Lisandro Medina (a la manera de Borges)»

Abulgualid Muhámmad ibn-Yussuf, heresiarca siriaco del siglo VI, en sus comentarios apócrifos sobre El libro de los Reyes, mantuvo (libro decimonono, apartado séptimo) que el doble tetrágono irregular no es una representación del Innominable, sino el propio Dios. Jorobado y falaz, no logró dirimir la secular disputa, pero sus cacofonías envilecieron considerablemente el problema (...).

\section{Texto III Agustín Fernández Mallo, «Argumentum ornithologicum»}

La existencia de Dios es un hecho innegable, aunque sólo sea por agotamiento lógico: hay quienes creen que Dios hizo al hombre a su imagen y semejanza, y hay quienes creen que fue el hombre quien hizo a Dios a su imagen y semejanza. El resultado es el mismo. El matemático ruso Georg Cantor (1895-1918) concibió una de las construcciones más importantes y extrañas de la historia matemática, los Números Transinfinitos, lo que no le impidió morir en la indigencia. A los 23 años de edad intuyó, y más tarde formalizó, que existen infinitos más grandes que otros infinitos, los que ordenó de menor mayor y denominó con la letra Aleph [...] del alfabeto hebreo [que simboliza a un pájaro en la máxima extensión del batir de sus alas; pero esto lo digo yo] (...)

Basta una comparación somera entre los textos de Borges, Sabato y Fernández Mallo para comprender por qué los enfoques estilísticos empleados por los críticos más tradicionales o formalistas se prestan más fácilmente para analizar la relación establecida entre el texto de Sabato y la obra de Borges. En efecto, con relativa sencillez detectamos los rasgos estilísticos reconcentrados que parecen aludir emblemáticamente a la imagen más conocida de la fraseología borgeana. El texto de Fernández Mallo, abiertamente postpoético, muestra por el contrario la ambigüedad de aquello que Benjamín Prado no sabía si calificar de «un[a] cita, tampoco un plagio (...) ni una alusión o referencia, pues hay cita literal». Algo todavía más marcado en este otro fragmento de El hacedor (de Borges) que bajo el título «El arrepentimiento de Heráclito» reproduce parte de las conversaciones registradas en las Torres Gemelas el 11 de septiembre de 2001: 


\section{Texto IV}

EED ME . CALL LIZ 8472935177 2001-09-11 15:52:01 Skytel [003330184] C SH/TONE 76102001-09-11 15:52:01 Skytel [005203329] A ALPHA 714792-5076 BREA LOANLINE \& RELATIONSHIPS CALLS HAVE BEEN ROUTED TO RANCHO OR EMERGENCY MESSAGE. ALL LINES HAVE BEEN TESTED. $<$ KD-MOYLE-12:50PT> 2001-09-11 15:52:01 Skytel [003252107] C ALPHA 714-792-5076 BREA LOANLINE \& RELATIONSHIPS CALLS HAVE BEEN ROUTED TO RANCHO OR EMERGENCY MESSAGE. ALL LINES HAVE BEEN TESTED $<$ KD-MOYLE-12:50PT $>(\ldots)$

La perplejidad de los críticos explica la reacción destemplada de la titular de los derechos de autor de Borges, pero también evidencia, a mi entender, las zonas de exclusión debidas a las definiciones propias de cada disciplina. En otras palabras, un crítico literario, un historiador de la literatura y un perito lingüista buscan cada uno las huellas textuales, los rasgos identificativos de los textos y autores. Pero, al diferir el objeto de estudio y sobre todo su finalidad, sus conclusiones o la significación que conceden a los resultados pueden divergir o incluso ser radicalmente opuestas.

\section{¿Es posible concebir métodos objetivos y científicos para identificar las} huellas y las apropiaciones literarias?

Una vez señalados los espacios de exclusión y la no correspondencia entre las definiciones centrales de cada disciplina, queda por resolver la cuestión sobre el estatus epistemológico de los modelos disciplinarios. Intentaré pues, en este segundo bloque, dar algunos elementos de reflexión a la pregunta: ${ }_{i} E s$ posible concebir métodos objetivos y científicos para identificar las huellas y las apropiaciones literarias? Para ello me serviré de una actividad habitual dentro de los estudios literarios y de la lingüística forense: la identificación de los autores textuales. La razón es que ambas subdisciplinas comparten principios y definiciones metodológicas, así como el hecho de que, a priori, ambas conceden una mayor importancia a las estrategias demostrativas y empíricas que a las estrategias persuasivas o interpretativas. Como resultado, este tipo de estudios parece disfrutar de un estatus más objetivo, incluso más científico, con respecto a las conclusiones de otras disciplinas como, por ejemplo, la Crítica literaria. Pero, ¿es esto realmente cierto? Para intentar una respuesta, haré un breve repaso de la metodología y las bases teóricas que la sustentan adoptadas por los distintos investigadores, así como su aplicación a un caso modélico: la identidad real tras el pseudónimo de 
Licenciado de Avellaneda, el infame autor del Quijote apócrifo, problema irresoluble desde hace cuatro siglos en las Letras hispanas.

La posibilidad efectiva de identificar a los productores de los mensajes lingüísticossebasa, antetodo, en implicaciones asumidas porlosinvestigadores sobre el lenguaje y la producción textual. Tanto para los investigadores literarios como para los peritos lingüistas, los locutores y autores textuales dejan huellas distintivas en los discursos, de la misma forma en que las huellas dactilares o el código genético permiten la identificación por encima de dudas razonables. La construcción de un corpus y unos protocolos contrastivos es, por consiguiente, el elemento clave a la hora de poder determinar los idiolectos de los autores posibles de lo que se conoce como un texto dubitado -se entiende por indubitados los que disfrutan de una autoría no cuestionada-. De este modo, el idiolecto resulta de la sustracción de los rasgos aislados en los textos indubitados (estilema) confrontados al corpus (para obtener su rareza o probabilidad) y confirmados o contrastados por los propios rasgos idiolectales, gramaticales y estilísticos del texto dubitado. Es aceptado comúnmente, por lo demás, que el análisis de los textos aporta claves sobre aspectos tales como la edad, el género, la etnicidad, la raza, el dialecto, el grado de educación, la creencia religiosa o incluso la ideología política y la profesión del escritor o hablante.

Estas implicaciones sobre el Lenguaje y la Escritura, comunes a la identificación estrictamente filológica y a la estilística forense -por otra parte también compartidas por otros paradigmas formalistas- pueden ser divididas a grandes rasgos en tres grandes grupos:

1. Las implicaciones que conciernen al proceso de creación.

a. La obra es el producto de un trabajo y de una voluntad.

b. Las obras presentan huellas lingüísticas de su productor, rasgos en principio singulares e identificables.

c. La originalidad (la diferencia frente a otras obras) es medible cuantitativa y cualitativamente.

2. Los axiomas que conciernen a la figural función del autor:

a. el autor es definido como un campo de coherencia conceptual o teórica;

b. el autor es considerado como unidad estilística y de habla. La información que podemos obtener a través del mero análisis de las formas verbales

c. el autor es un cronotopo, un momento histórico definido y punto de encuentro de cierto número de acontecimientos. 
3. Consideraciones diversas sobre el lenguaje, heredadas de distintas disciplinas asociadas a los estudios lingüísticos, fundamentalmente nociones básicas del paradigma actual (así la noción de «horizonte de expectativas», "cambio lingüístico», «norma», etc.).

Todos estos apriorismos son en sí mismos indemostrables como lo muestra su aplicación en casos concretos. Si observamos el siguiente cuadro, muchos son los nombres que han sido propuestos como verdadera identidad del Licenciado Avellaneda, pero debajo de todas las propuestas subyacen estas implicaciones que acabo de enumerar, que no necesitan demostración, puesto que forman parte de los presupuestos disciplinarios. Si observamos el cuadro con más atención, no es descabellado aventurar que la irrupción de las Nuevas Tecnologías a finales del siglo pasado añadió un mayor atractivo a las estrategias formalistas, más cercanas a la materialidad de la escritura que a la explicación puramente literaria o estética.

\begin{tabular}{|l|c|c|l|}
\hline \multicolumn{1}{|c|}{ Identidades supuestas del Licenciado Avellaneda } \\
\hline \multicolumn{1}{|c|}{ Investigador } & Año & \multicolumn{1}{c|}{ Autor supuesto } & \multicolumn{1}{c|}{ Razones } \\
\hline VV.AA. & $\begin{array}{c}\text { Antes de } \\
1970\end{array}$ & $\begin{array}{l}\text { Hermanos Argensola, } \\
\text { Mateo Alemán, Fray Andrés Pérez, } \\
\text { Juan Martí, Francisco de Quevedo, } \\
\text { Cristóbal de Fonseca, Guillén de Castro, } \\
\text { Castillo de Solórzano, etc }\end{array}$ & Extratextuales (literarias) \\
\hline José López Navío & $* 2005$ & Tirso de Molina & Extratextuales (literarias) \\
\hline Daniel Eisenberg & 1991 & Lope de Vega & $\begin{array}{l}\text { Extratextuales (literarias): } \\
\text { Animadversión hacia Cervantes }\end{array}$ \\
\hline $\begin{array}{l}\text { Martín de Riquer/ } \\
\text { Alfonso Martín Jiménez/ } \\
\text { Juan Antonio Frago Gracia }\end{array}$ & $2001 /$ & Jerónimo de Passamonte & $\begin{array}{l}\text { Coincidencias textuales/ } \\
\text { estilemas similares/ recurrencia } \\
\text { de hápax }\end{array}$ \\
\hline Antonio Sánchez Portero & 2006 & Pedro Liñán de Riaza & $\begin{array}{l}\text { Extratextuales (literarias): } \\
\text { Animadversión hacia Cervantes }\end{array}$ \\
\hline Enrique Suárez Figaredo & 2008 & Cristóbal Suárez de Figueroa & $\begin{array}{l}\text { Coincidencias textuales/ } \\
\text { estilemas similares/ } \\
\text { recurrencia de hápax }\end{array}$ \\
\hline $\begin{array}{l}\text { Alfredo Rodríguez López- } \\
\text { Vázquez }\end{array}$ & 2011 & $\begin{array}{l}\text { Coincidencias textuales/ estile- } \\
\text { mas similares/ recurrencia de } \\
\text { hápax/ «huellas de lecturas» }\end{array}$ \\
\hline
\end{tabular}

La manera en que los filólogos y los peritos lingüistas analizan los textos es grosso modo idéntica en lo que respecta al tratamiento automatizado de los textos. Por comodidad, utilizo aquí los sistemas informáticos de detección automática del plagio -especialmente a partir de los trabajos de Alberto Barrón Cedeño, ingeniero informático especializado en la cuestión-, así como la teoría de la Lingüística forense a través de dos importantes compendios colectivos, uno anglosajón y otro español -The 
Routledge Handbook of Forensic Lingustics, editado por Malcolm Coulthard y Alison Johnson, y Lingüistica forense, lengua y derecho. Conceptos, métodos $y$ aplicaciones, editado por María Teresa Turell i Julià - puesto que esta disciplina ha llevado a cabo recientemente una amplia discusión sobre muchos de los procedimientos heurísticos, compartidos por la metodología "literaria". Así, donde los autores hablan de «texto fraudulento» o de "plagio», los investigadores literarios podrán leer «intertextualidad», «alusión», «cita», "préstamo», «huella», etc.

Los programadores y diseñadores de los sistemas de detección parten de premisas lógicas que determinan la forma que adoptan los algoritmos, valores y parámetros que entrarán en juego a la hora de cotejar el texto dubitado y los textos del corpus de referencia. La forma silogística que adoptan es grosso modo la siguiente:

Sean $s$ un documento sospechoso y $D$ un conjunto de documentos de referencia, el objetivo de la detección automática de plagio es encontrar aquel documento $d \in D$ que haya sido utilizado como fuente para obtener el documento $s$, el cual presumiblemente es un caso de plagio. Dicha búsqueda puede llevarse a un nivel más específico: Sea $s_{i} \in s$ un fragmento plagiado, el objetivo es encontrar aquel fragmento $d_{j} \in \mathrm{d}$ tal que $d_{j}$ es la fuente del fragmento plagiado $s_{i}$ (Barrón Cedeño 2008: 5).

Esta indagación lógica adquiere la forma de una ecuación matemática que servirá de base para el conjunto de los parámetros que clasificarán el grado de dependencia textual entre el texto dubitado $s$ y el corpus $D$. Se denomina $R(<$ Resemblance $)$ esta función de similitud textual entre el conjunto de segmentos de un texto $\mathrm{S}(\mathrm{A})$ y los de un texto $\mathrm{S}(\mathrm{B})$, valor que aumentará cuanto más segmentos compartan A y B, indicando una mayor probabilidad de dependencia (Lyon, Malcolm Y Dickerson 2001:118-125):

$$
R=\frac{|S(A) \cap S(B)|}{|S(A) \cup S(B)|}
$$

En las operaciones de cotejo de los sistemas de detección con referencia no todos los segmentos son considerados igualmente pertinentes o significativos. Suele entenderse que las preposiciones, artículos o pronombres, palabras extremadamente frecuentes y poco susceptibles de marcar la variación estilística, pueden falsear los análisis y ocultar la importancia de otras repeticiones. Asimismo, el empleo de las estrategias de inserción, supresión y reescritura puede ocultar patrones de repetición, glosa o composición resultantes de una presumible dependencia textual. Por ello, los programadores y analistas suelen preferir lo que se ha denominado 
el sistema de detección por $\mathrm{N}$-gramas, segmentos que varían de una a varias palabras, evitando de este modo la alteración del orden de las palabras o las desviaciones producidas por la inserción o supresión de palabras. De los sistemas de detección que tienen en cuenta distintas variables estilométricas, el coeficiente de similitud $\mathrm{R}$ u otros valores, se dice que emplean una lógica difusa (fuzzy logic) (Wools 2010: 579).

Los sistemas de detección con referencia pueden ser combinados con sistemas de detección intrínsecos que se centran en la detección de los segmentos más susceptibles de ser ajenos dentro del documento dubitado. Estas estrategias intrínsecas se basan en fórmulas estadísticas empleadas en Sociolingüística para separar los registros y variedades del Habla. Entre estos parámetros se encuentra el coeficiente de legibilidad que otorga valores en función de la longitud y el grado de subordinación sintáctica medios, la longitud media de las lexías (que supuestamente refleja el nivel cultural de los autores/ locutores), así como la riqueza léxica relativa que tiene en cuenta la existencia de hápax (ocurrencias únicas) y su frecuencia relativa (la recurrencia y un índice bajo de ocurrencias singulares es interpretado como un indicio de pobreza léxica relativa). Este, por ejemplo, es uno de los motivos esgrimidos por Enrique Suárez Figaredo para sostener la candidatura de Cristóbal Suárez de Figueroa, puesto que hay expresiones que sólo han aparecido por el momento en el Quijote apócrifo y en la Constante Amarilis.

Otras categorías discursivas susceptibles de producir hápax y que también son destacadas en los análisis forenses conciernen a los errores ortográficos, tipográficos y gramaticales. Su repetición continuada o en contextos similares puede llegar a ser interpretada como una evidencia determinante de dependencia textual, a pesar de que deban ser confrontados a la Norma vigente en cada momento histórico (un error ortográfico puede ser muy frecuente en un periodo determinado). Esta es una de las evidencias textuales por las que Martín de Riquer y Juan Antonio Fragua atribuyen la paternidad a Jerónimo de Passamonte, puesto que, en su opinión, el texto de Avellaneda presenta aragonesismos y una supuesta pobreza léxica explicable por las pocas letras del soldado aragonés.

A fin de cuentas, a pesar de la aparente objetividad de las formulaciones lógico-matemáticas seguimos sin tener una identificación indiscutible para el licenciado Avellaneda. Los estudios se suceden, pero a pesar de las estadísticas esgrimidas, las frecuencias léxicas o los hápax detectados, no parece que la resolución del enigma esté más cerca que antes de la 
utilización masiva de las nuevas Tecnologías. Cada investigador otorga un peso diferente a los resultados, que en sí mismos pueden ser objetivos, pero cuya interpretación resulta, en definitiva, siempre subjetiva.

Otras debilidades responden a las limitaciones tecnológicas. Así, un obstáculo estrictamente técnico es la posibilidad de la sinonimia o equivalencia en los lenguajes naturales. Un ordenador nunca podrá comprender ni un calambur, ni un eufemismo, la ironía o un juego de palabras; es decir, las relaciones de sentido, más allá de la forma, entre los textos. Asimismo, otra objeción que suscita a menudo este tipo de trabajos pone en duda la validez de las elecciones subjetivas que los peritos realizan, especialmente en la elaboración de corpus y la identificación de los segmentos anormales, argumentos reiterados por los últimos estudiosos de Avellaneda. Numerosos investigadores, aunque admiten el problema y reclaman cautela y discreción en las inferencias obtenidas a partir de la confrontación textual, se muestran «optimistas» y consideran que la digitalización continua generará corpus cada vez más extensos y fiables, que permitirán establecer con mayor fiabilidad la frecuencia relativa frente a una Norma que podrá al fin ser abstraída.

No obstante, es posible realizar otra crítica de índole metodológica sobre los corpus empleados por la mayoría de los estudiosos de Avellaneda para establecer la Norma literaria y poder realizar las confrontaciones oportunas. Muchos investigadores han utilizado el CORDE (corpus histórico) o el CREA (español actual), ambos de la RAE, cuya fiabilidad continúa siendo limitada, puesto que recogen muestras muy parciales de textos juzgados a priori como significativos (por el principio de autoridad o de representatividad percibida). En mi opinión, incluso si estos corpus fueran ampliados y los criterios de inclusión mejorados, nunca dejarían de ofrecer resultados aproximativos y no una ratio indiscutible a partir de la cual sería posible establecer sin lugar a dudas la normatividad de una ocurrencia determinada.

Termino con una conclusión sujeta a debate y con varias preguntas. La matematización de las disciplinas filológicas las hace indudablemente más sofisticadas, pero no por ello más científicas 5 . En última instancia, los

5 Resulta significativo que sigan siendo válidas hoy día las mismas objeciones que suscitaron ya en 1973, en autores como Stanley Fish (1980: 99-111), los primeros intentos de aplicar el tratamiento automatizado del lenguaje a la Estilística. Es más, recientemente un estudio afirmaba nada más y nada menos que un «modelo simple de evolución estilística» para la literatura anglosajona desde los tiempos de Shakespeare hasta el siglo XIX a partir del estudio distribucional de tres centenares de términos y lexías complejas (Hughes, Fotia, Krakauer, Rockmor 2012). No es que los contrargumentos de Fish hayan sido 
argumentos extratextuales (por ejemplo, coherencia estética o ideológica) parecen tener un peso comparable a los indicios estrictamente textuales. Algunas de las debilidades de los procedimientos automatizados radican en las definiciones de base, puesto que son en sí mismas infalsables. Debemos, por consiguiente, reconocer que existen preguntas a las que ni la Lingüística ni la Teoría literaria pueden dar respuestas plenamente satisfactorias, es decir, fuera de toda discusión y aceptadas consensuadamente por la comunidad científica como definitivas. Por ejemplo, si se asume que un autor guarda siempre una coherencia idiomática, ¿qué hacer entonces con el pastiche de Sabato? (Texto II), ¿realmente se puede excluir que un escritor pueda suplantar completamente la identidad literaria (estilema) de otro? O cambiando de ángulo: ¿Cómo distinguir los préstamos de las apropiaciones? O más difícil todavía: ¿Cómo formalizar una identidad textual como la de Fernández Mallo (Texto III y IV), creada a partir de una poética de apropiación y referencia ilimitadas?

\section{Bibliografía}

Barrón Cedeño, Luis Alberto, 2008, Detección automática de plagio en texto. Tesis de Máster, Departamento de Sistemas Informáticos y Computación, Dirigida por Paolo Rosso, Valencia, Universidad de Valencia.

Coulthard, Malcolm, Johnson, Alison (eds.), 2010, The Routledge Handbook of Forensic Linguistics, London, New York, Routledge.

Fernández Mallo, Agustín, 2011, El hacedor (de Borges). Remake, Madrid, Alfaguara. Fish, Stanley, 1980, Is There a Text in This Class? The Authority of Interpretive Communities, Cambridge (Massachusetts), Harvard University Press.

Foucault, Michel, 2001, «Qu'est-ce qu'un auteur? [1969]» En Dits et Écrits I. 19541975, Paris, Gallimard, p. 817-849.

Frago García, Juan Antonio, 2005, El Quijote apócrifo y Pasamonte, Madrid, Gredos. Grafton Anthony, 1990, Forgers and Critics. Creativity and Duplicity in Western Scholarship, Princeton (New Jersey), Princeton University Press.

Hughes, Fotia, Krakauer y Rockmor, abril 2012, «Quantitative patterns of stylistic influence in the evolution of literature», en Proceedings of the National Academy of Sciences of the United States of America.

rebatidos, han sido sencillamente ignorados. Algo característico en los defensores de la matematización del estilo, de formación fundamentalmente lingüística, que simplemente hacen caso omiso de las advertencias metodológicas y heurísticas sobre la posibilidad misma de formalizar la norma lingüística o literaria. 
Lyon, Caroline, James, Malcolm y Dickerson, Bob, 2001, "Detecting Short Passages of Similar Text in Large Document Collections," en Proceedings of the 2001 Conference on Empirical Methods in Natural Language Processing, Ithaca, Cornell University.

Perromat Augustín, Kevin, julio 2011, «Plagiarismo: ¿estética o movimiento contemporáneo?», $452^{\circ} \mathrm{F}$, Revista de teoría de la literatura y literatura comparada, no 5, Barcelona, Universitat Autònoma de Barcelona, p. 115-127.

Perromat Augustín, Kevin, 2010, El plagio en las literaturas hispánicas: Historia, Teoría y Práctica, tesis doctoral bajo la dirección de Dña, Milagros Ezquerro, Universidad Paris-Sorbona Paris IV, http://www.e-sorbonne.fr/sites/www.esorbonne.fr/files/theses/These_LePlagiat_KevinPERROMAT.pdf

Rubio Tovar, Joaquín, 2004, La vieja diosa. De la Filología a la Posmodernidad. (Algunas notas sobre la evolución de los estudios literarios), Alcalá de Henares (Madrid), Centro de Estudios Cervantinos.

Rodríguez López-Vázquez, Alfredo, 2011, «El Quijote de Avellaneda: nuevos índices de atribución a José de Villaviciosa» en LEMIR. Revista de Literatura Española Medieval y del Renacimiento, $n^{\circ}$ 15, Alicante: Universidad de Alicante, p. 261-280. Sabato, Ernesto, «El tetrágono de Lisandro Medina (a la manera de Borges)», Suplemento «Babelia» de El País, Madrid, 4 de marzo de 1995.

Senís Fernández, Juan, 2001, «No es novísimo todo el culturalismo que reluce. Una aproximación a la poesía de Benjamín Prado,» Hesperia. Anuario de filología hispánica (Universidad de Vigo), nº 4, p. 137-159.

Suárez Figaredo, Enrique, 2011, «Sobre la atribución del Quijote apócrifo a José de Villaviciosa» en LEMIR, Revista de Literatura Española Medieval y del Renacimiento, $\mathrm{n}^{\circ}$ 15, Alicante, Universidad de Alicante, p. 261-280.

Turell I Julià, María Teresa, (ed.), 2005, Lingüística forense, lengua, derecho. Conceptos, métodos y aplicaciones, Barcelona, Institut Universitari de Lingüística Aplicada, Universitat Pompeu Fabra. 